\title{
The chemical composition of the circumstellar envelopes around yellow hypergiant stars ${ }^{\star} \star \star \star$
}

\author{
G. Quintana-Lacaci ${ }^{1}$, V. Bujarrabal ${ }^{1}$, A. Castro-Carrizo ${ }^{2}$, and J. Alcolea ${ }^{3}$ \\ 1 Observatorio Astronómico Nacional (IGN), Apdo. 112, 28803 Alcalá de Henares, Spain \\ e-mail: [g.quintana;v.bujarrabal]@oan.es \\ 2 Institut de RadioAstronomie Millimétrique, 300 rue de la Piscine, 38406 Saint Martin d'Hères, France \\ e-mail: ccarrizo@iram.fr \\ 3 Observatorio Astronómico Nacional (IGN), Alfonso XII N³, 28014 Madrid, Spain \\ e-mail: j.alcolea@oan.es
}

Received 6 February 2007 / Accepted 24 April 2007

\section{ABSTRACT}

\begin{abstract}
Context. The yellow hypergiant stars (YHGs) are extremely luminous and massive objects whose general properties are poorly known. Only two of this kind of star show massive circumstellar envelopes, IRC +10420 and AFGL 2343.

Aims. We aim to study the chemistry of the circumstellar envelopes around these two sources, by comparison with well known AGB stars and protoplanetary nebulae. We also estimate the abundances of the observed molecular species.

Methods. We have performed single-dish observations of different transitions for twelve molecular species. We have compared the ratio of the intensities of the molecular transitions and of the estimated abundances in AFGL 2343 and IRC +10420 with those in O-rich and C-rich AGB stars and protoplanetary nebulae.

Results. Both YHGs, AFGL 2343, and IRC +10420, have been found to have an O-rich chemistry similar to that in O-rich AGB stars, though for AFGL 2343 the emission of most molecules compared with ${ }^{13} \mathrm{CO}$ lines is relatively weak. Clear differences with the other evolved sources appear when we compare the line intensity corrected for distance and the profile widths which are, respectively, very intense and very wide in YHGs. The abundances obtained for IRC +10420 agree with those found in AGB stars, but in general those found in AFGL 2343, except for ${ }^{13} \mathrm{CO}$, are too low. This apparently low molecular abundance in AFGL 2343 could be due to the fact that these molecules are present only in an inner region of the shell where the mass is relatively low.
\end{abstract}

Key words. stars: circumstellar matter - stars: supergiants - stars: AGB and post-AGB - radio lines: stars stars: individual: IRC +10420 - stars: individual: AFGL 2343

\section{Introduction}

The Yellow hypergiants stars (YHGs) are among the most luminous $\left(5.3 \leq \log L\left[L_{\odot}\right] \leq 5.9\right)$ and massive $\left(M_{\text {init }} \sim 20 M_{\odot}\right)$ stars (see, as general references, de Jager 1998; Jones et al. 1993; Humphreys 1991). These objects are thought to be post-red supergiants evolving bluewards in the HR diagram, but the details of such an evolution are still unknown. In at least a few of them, the stellar temperature is rapidly increasing. For instance, the spectral type of the hypergiant IRC +10420 has changed from F8Ia to A5Ia in just 20 yr (Klochkova et al. 1997; Oudmaijer et al. 1996; Oudmaijer 1998). Humphreys et al. (2002) showed, however, that the wind in this source is optically thick, suggesting that the apparent spectral type changes are due to variations in the wind rather than to interior evolution.

Although it is thought that, during the red and yellow phases, these heavy stars eject as much as one half of their initial mass (e.g. Maeder \& Meynet 1988; de Jager 1998), only two YHGs, IRC +10420 (=IRAS 19244+1115) and AFGL 2343 $(=$ IRAS $19114+0002=$ HD 179821) are known to have very heavy circumstellar envelopes (CSEs). Those CSEs were

\footnotetext{
* Based on observations carried out with the IRAM Pico Veleta $30 \mathrm{~m}$ telescope. IRAM is supported by INSU/CNRS (France), MPG (Germany) and IGN (Spain).

$\star \star$ Appendix A is only available in electronic form at http://www. aanda.org
}

detected in molecular line emission, dust-scattered light and IR emission (see Hawkins et al. 1995; Meixner et al. 1999; Bujarrabal et al. 1992; Humphreys et al. 1997; Neri et al. 1998; Bujarrabal et al. 2001; Castro-Carrizo et al. 2001).

Recent results from Castro-Carrizo et al. (2007) show that these circumstellar envelopes have several solar masses, very high expansion velocities $\left(\sim 35 \mathrm{~km} \mathrm{~s}^{-1}\right)$, and that such an environment was formed in the last $\sim 6000 \mathrm{yr}$. The envelope properties there found are compatible with a mass loss driven by radiation pressure. However, in the case of IRC +10420 the existence of infalling material suggests that other processes are also present in the inner parts of the envelope (Humphreys et al. 2002). Other properties of these molecular shells, like their chemical composition, have not been well studied.

This paper is devoted to the study of the chemistry in the envelopes around IRC +10420 and AFGL 2343. We have observed rotational lines of several molecules and, in particular, we compare the line intensities and molecular abundances found in these objects with those of AGB CSEs and protoplanetary nebulae (PPNe).

Both IRC +10420 and AFGL 2343 have very probably O-rich chemistry, in view of their $\mathrm{OH}$ maser emission (Likkel 1989; Reid et al. 1979; among evolved objects, only O-rich stars are found to emit in $\mathrm{OH}$ masers), silicate-rich circumstellar grains (e.g. Molster et al. 2002) and C-poor atmospheric composition (Klochkova et al. 1997; Thévenin et al. 2000). 
Table 1. Program stars. References: (H) Hipparcos, (1) Jones et al. (1993), (2) Bujarrabal et al. (1994a), (3) Skinner et al. (1998), (4) Jourdain de Mouizon et al. (1990).

\begin{tabular}{lccccc}
\hline \hline Name & $\begin{array}{c}\text { Observation } \\
\alpha(2000)\end{array}$ & $\begin{array}{c}\text { coordinates } \\
\beta(2000)\end{array}$ & $\begin{array}{c}V_{\text {LSR }} \\
\left(\mathrm{km} \mathrm{s}^{-1}\right)\end{array}$ & $\begin{array}{c}D \\
(\mathrm{kpc})\end{array}$ & Comments \\
AFGL 2343 & 191358.6 & 000732 & 98 & $5.6^{\mathrm{H}}$ & Yellow hypergiant \\
IRC + 10420 & 192648.0 & 112117 & 76 & $5^{1}$ & Yellow hypergiant \\
\hline IRC +10216 & 094757.4 & 131644 & -26 & $0.2^{2}$ & C-rich AGB star \\
CRL 2688 & 210218.8 & 364138 & -35 & $1.2^{3}$ & C-rich PPN \\
NGC 7027 & 210701.6 & 421410 & 26 & $1^{4}$ & C-rich Young PN \\
RX Boo & 142411.6 & 254213 & -2 & $0.2^{2}$ & O-rich AGB star \\
TX Cam & 050050.4 & 561053 & 9 & $0.35^{2}$ & O-rich AGB star \\
\hline
\end{tabular}

Castro-Carrizo et al. (2007) argued that AFGL 2343 was a YHG rather than a PPN as proposed by Josselin \& Lèbre (2001). As we will see, our molecular data confirm this, since the molecular line properties of these objects are quite similar and significantly different from those usual in both PPNe and AGB stars. On the other hand, the nature of IRC +10420 is well known (see Jones et al. 1993; Oudmaijer et al. 1996). Our data will be used, in general, to determine the main chemical properties of the envelopes surrounding IRC +10420 and AFGL 2343.

\section{Observations}

We have used the IRAM 30 m telescope, at Pico Veleta (Spain), to observe mm-wave molecular lines in the yellow hypergiants IRC +10420 and AFGL 2343. The observed lines are:

- In the 3 mm band: $\mathrm{C}^{18} \mathrm{O} J=1-0, \mathrm{HCN} J=1-0, \mathrm{H}^{13} \mathrm{CN} J=$ $1-0, \mathrm{SiO} J=2-1,{ }^{29} \mathrm{SiO} J=2-1, \mathrm{CS} J=2-1$, CN $N=1-0$, $\mathrm{SiS} J=5-4$, SO $J_{K}=2_{2}-1_{1}, \mathrm{HC}_{3} \mathrm{~N} J=10-9$, HNC $J=1-0$, and $\mathrm{HCO}^{+} J=1-0$.

- In the 2 mm band: $\mathrm{SiO} J=3-2$ and CS $J=3-2$.

- In the $1 \mathrm{~mm}$ band: $\mathrm{C}^{18} \mathrm{O} J=2-1, \mathrm{HCN} J=3-2$, SiO $J=$ $5-4,{ }^{29} \mathrm{SiO} J=5-4, \mathrm{CS} J=5-4, \mathrm{CN} N=2-1$, SiS $J=$ $15-14$, $\mathrm{HNC} J=3-2$, and $\mathrm{HCO}^{+} J=3-2$.

In order to compare the line properties in these sources with those in other evolved circumstellar envelopes, we have also observed the O-rich AGB stars RX Boo and TX Cam, the C-rich AGB star IRC +10216, the C-rich PPN CRL 2688, and the young C-rich PN NGC 7027.

The coordinates of the star and some stellar properties of the program stars are summarized in Table 1 . In IRC +10216 and CRL 2688, that are intense emitters, some other lines also appeared within the simultaneous receiver bands of the above lines.

Our observations were performed in June 2000. SIS 3, 2, and $1 \mathrm{~mm}$ receiver bands were used, often simultaneously. The receivers were tuned always in SSB mode. From frequent pointing measurements, we expect errors $\sim 3^{\prime \prime}$. The spatial resolution is $12-13^{\prime \prime}$ at $1.3 \mathrm{~mm}$ (taking into account the effects of pointing errors) and 22-26" at $3 \mathrm{~mm}$. Beam efficiencies ranged between $\sim 0.75$ at $3 \mathrm{~mm}$ wavelength and $\sim 0.5$ at $1 \mathrm{~mm}$.

The data presented here are calibrated in units of main beam Rayleigh-Jeans-equivalent antenna temperature, $T_{\mathrm{mb}}$, using the chopper-wheel method by observing hot (ambient) and cold loads (liquid nitrogen). The atmospheric conditions were good, with zenith opacities $\lesssim 0.2$ at $\lambda=1 \mathrm{~mm}$. In addition, some results were compared with previous observations by Bachiller et al. (1997a,b), Bujarrabal et al. (1994a), and the catalog of standard line intensities for the $30 \mathrm{~m}$ telescope (Mauersberger et al. 1989) in order to improve calibration. From the comparison between our different spectra and with respect with previous data, we expect calibration uncertainties of about $20 \%$.

The detected spectra in our main sources, IRC +10420 and AFGL 2343, are shown in Figs. 1 and 2. Note that, in the case of CN $N=2-1$, only the two main groups of line components were observed, corresponding to $J=5 / 2-3 / 2$ and $J=3 / 2-1 / 2$. In Figs. A.1-A.7 (in electronic version only) we present the spectra of the other observed sources: CRL 2688, NGC 7027, RX Boo, TX Cam and IRC +10216 . As baseline profile, only straight lines were subtracted.

\section{Observational results}

We have obtained molecular line data of two YHGs, IRC +10420 and AFGL 2343, as well as for other sources, see Table 1 .

In Table 2 we show a summary of the new observational results: peak intensity $(\mathrm{K})$, rms noise and profile area $\left(\mathrm{K} \mathrm{km} \mathrm{s}^{-1}\right)$. These parameters were in most cases calculated for a spectral resolution of $1 \mathrm{MHz}$ for the $3 \mathrm{~mm}$ lines and of $2 \mathrm{MHz}$ for the $2 \mathrm{~mm}$ and $1 \mathrm{~mm}$ lines. The actual observations were performed with various spectrometers, whose resolutions were degraded if necessary to calculate these parameters. The upper limits are 5- $\sigma$ level, calculated as follows: Integrated Area $<5 \sigma \Delta V / \sqrt{N}$, being $\sigma$ the rms noise for the observed spectrum, $\Delta V$ the equivalent velocity width, and $N$ the number of channels within this velocity width. For the calculation of $\Delta V$ in the case of $\mathrm{CN}$, we take the equivalent width of $\mathrm{CN}$ in IRC +10216 (Bachiller et al. $1997 \mathrm{~b}$ ) as the sum of the areas of all the hyperfine transitions divided by its $T_{\mathrm{mb}}$. This CN equivalent width of IRC +10216 is renormalized multiplying it by the ratio between $\Delta V$ for ${ }^{13} \mathrm{CO}$ $J=2-1$ in the star for which the limit is being calculated and $\Delta V$ for ${ }^{13} \mathrm{CO}$ in IRC +10216 .

We have also considered the observations of various molecules from Bujarrabal et al. (1994a) and Bujarrabal et al. (1992), ${ }^{13} \mathrm{CO} J=1-0$ and $2-1$ data from Bujarrabal et al. (2001), CN $N=1-0$ and $2-1$ data from Bachiller et al. (1997b), and $\mathrm{HCO}^{+} J=1-0$ data from Bachiller et al. (1997a), Sánchez Contreras el al. (1997), and Lucas \& Guélin (1990). Note the interest of using data obtained with the same telescope, the IRAM $30 \mathrm{~m}$ dish, in order to better interpret the line ratios. Taking into account data from the sources in these papers, the total sample of objects which data are used in our analysis, together with the YHGs, is:

O-rich AGB stars and red supergiant stars (RSGs): RX Boo, TX Cam, R Cnc, RS Cnc, VY CMa, R Cas, $o$ Cet, NML Cyg, W Hya, R Leo, VX Sgr, IK Tau, RT Vir, IRC-10529, $\mathrm{IRC}+10011, \mathrm{OH} 26.5+0.6, \mathrm{OH} 44.8-2.3$. 


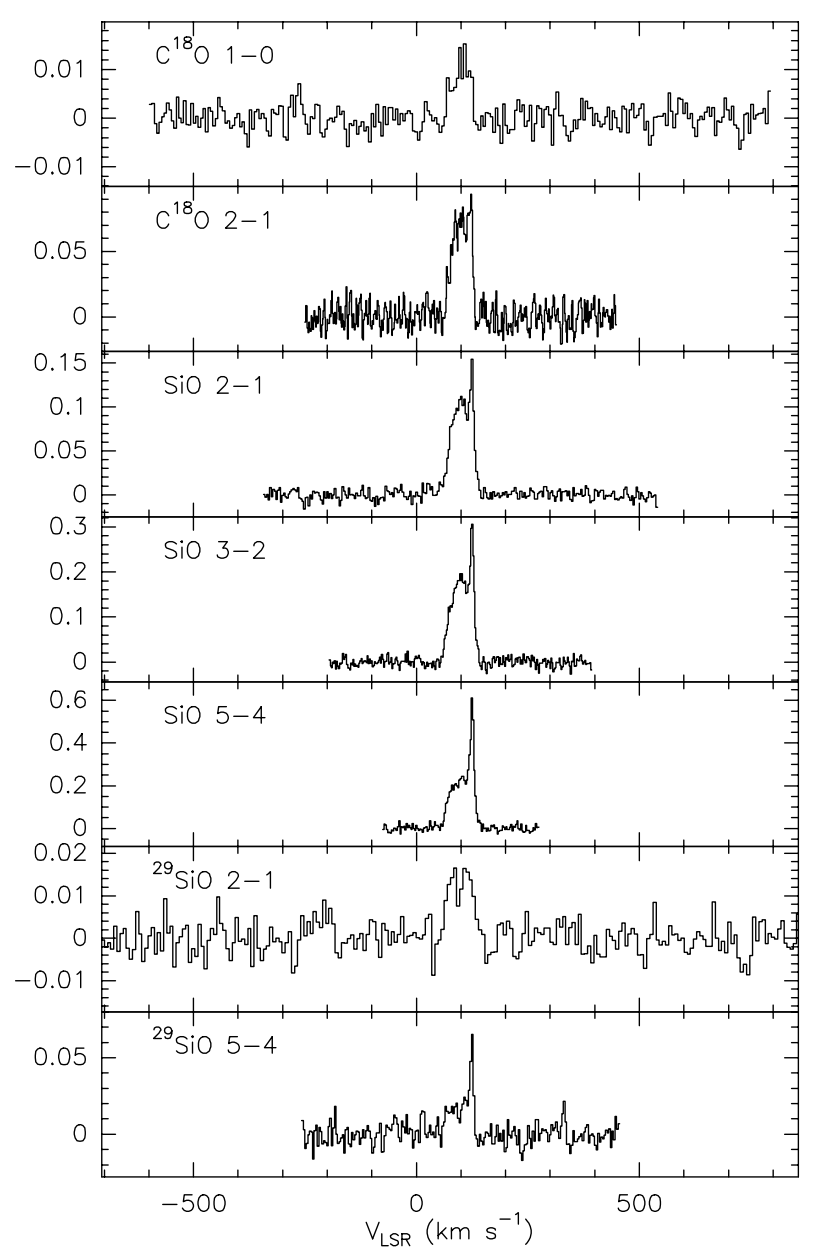

AFGL 2343

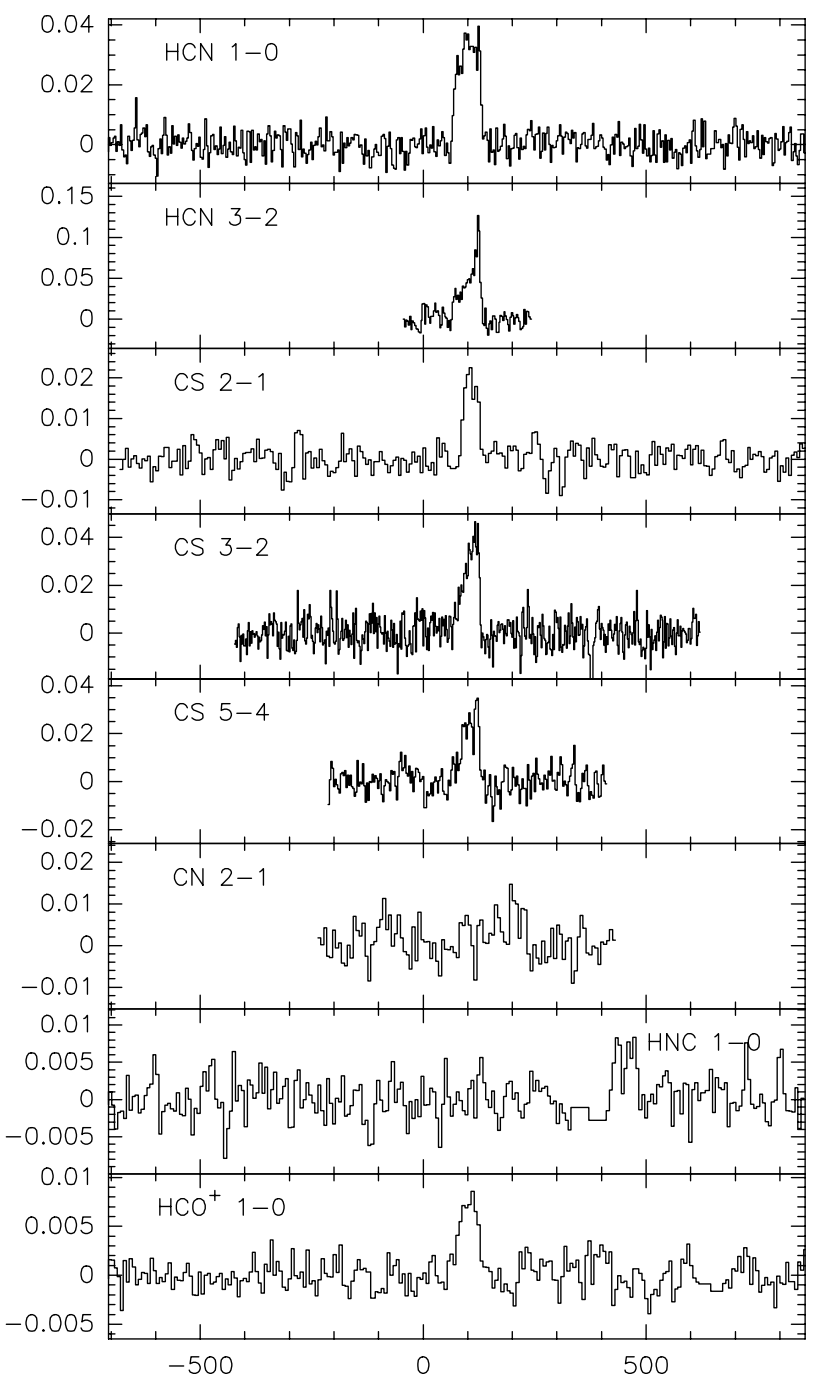

Fig. 1. Observed spectra towards AFGL 2343. The intensity scale is given in units of main-beam brightness temperature (K).

C-rich AGB stars: LP And, UU Aur, U Cam, Y CVn, S Cep, V Cyg, UX Dra, V Hya, CIT-6, CRL 865, CRL 3068, IRC10236, IRC +10216, IRC +20370, IRC +30374, IRC +60144.

O-rich PPNe: $\mathrm{OH}$ 17.7-2.0, OH 231.8+4.2, IRAS 17436+5003.

C-rich PPNe: CRL 618, CRL 2688, NGC 7027, IRAS 07134+ 1005, IRAS 19500-1709.

Note that the RSGs VY CMa, NML Cyg, and VX Sgr are also included, but very few data on them are available. From the point of view of the line intensity ratio comparison, and also due to the lack of data for these objects, both RSGs and O-rich AGBs behave in a similar way. Also, NGC 7027, a C-rich young $\mathrm{PN}$, is considered in the following discussion as a PPN.

\subsection{Line intensity comparison}

In order to compare the molecular emission in YHGs with that of other CSEs, we have represented in Figs. 3 to 5 integrated intensities of several pairs of relevant lines. Open symbols represent O-rich objects, and filled symbols C-rich objects. Squares and triangles respectively represent detections and limits for the AGB stars (AGBs). The RSGs are plotted as O-rich AGBs with thicker symbols. Four-peak asterisks and three-peak asterisks represent, respectively, detections and limits for PPNe. These polygons with three vertices have one of them pointing leftwards or downwards, indicating for which transition we have a limit. Objects with limits for the two represented lines are not plotted. For the YHGs, a five-peak open asterisk represents AFGL 2343, a six-peak asterisk is used for IRC +10420 , and the limits are represented by an arrow from the center of the symbol. To make the comparison easier with the other objects, straight lines (hereafter average lines) represent the points with a ratio equal to the geometric average of the profile area ratios for the two YHGs.

In Fig. 3 we show ratios between integrated line intensities for ${ }^{13} \mathrm{CO}$ and other abundant molecules. In Figs. 4 and 5 we show line ratios between pairs of molecules other than ${ }^{13}$ CO. In Fig. 3 we can see that, in general, AFGL 2343 and IRC +10420 lay in significantly different regions of the diagrams. For IRC +10420 these line ratios are comparable to these typical in O-rich AGB stars. For AFGL 2343, however, they are weaker by factors ranging between $\sim 2$ and 10 . On the other hand, in Figs. 4 and 5 both YHGs fall very close to the average line and to the region of the diagrams occupied by O-rich AGB stars, and their line ratios are clearly different from those of C-rich stars and PPNe. The situation of AFGL 2343 in Fig. 3 could be due to an overabundance in ${ }^{13} \mathrm{CO}$, an underabundance 

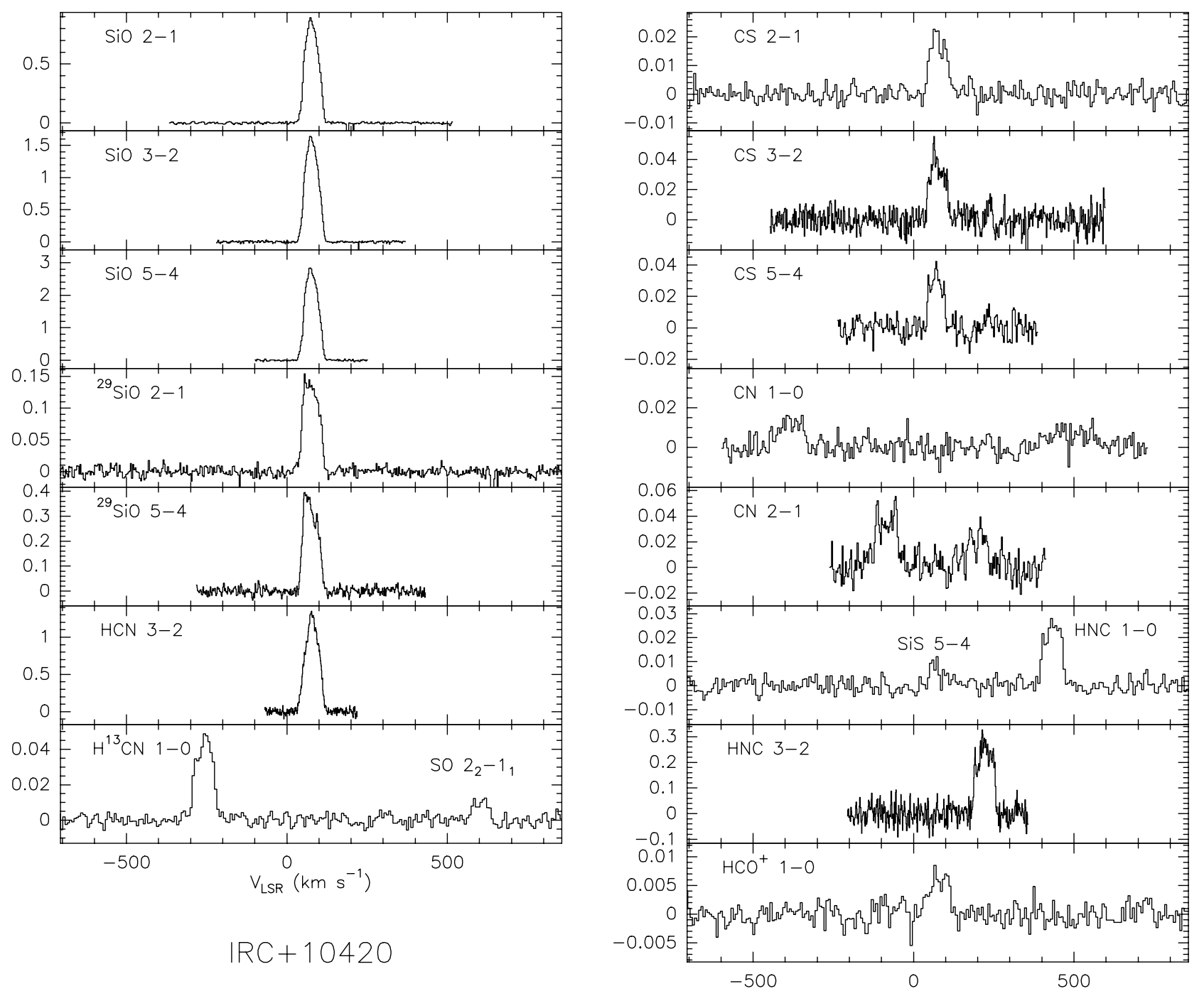

Fig. 2. Observed spectra towards IRC +10420 . The intensity scale is given in units of main-beam brightness temperature (K).

of the rest of the molecules, or to a small mass of the emitting regions for these molecules. Note that, for optically thin lines, the ratio of the profile areas depends on the abundance of the molecule and the mass of the emitting region. We can conclude from these diagrams that the YHGs show, as expected, O-rich chemistry similar to that of the O-rich AGB stars.

The relative weakness of most molecular lines with respect to ${ }^{13} \mathrm{CO}$ in AFGL 2343 is particularly noticeable for ${ }^{28} \mathrm{SiO}$, ${ }^{29} \mathrm{SiO}, \mathrm{HCN}, \mathrm{CN}$ and $\mathrm{HNC}$. The ${ }^{28} \mathrm{SiO} /{ }^{13} \mathrm{CO}$ intensity ratio in AFGL 2343 is similar to that of PPNe, and lower than those ratios obtained for AGBs, O-rich or C-rich. We also confirm the previously-reported low $\mathrm{SiO}$ intensity in PPNe.

The HCN intensity of AFGL 2343, relative to ${ }^{13} \mathrm{CO}$, lays under all O-rich AGBs. The $\mathrm{HNC} /{ }^{13} \mathrm{CO}$ and $\mathrm{CN} /{ }^{13} \mathrm{CO}$ line intensity ratios in this source are the lowest among all sources in our sample in which $\mathrm{HCN}$ or $\mathrm{CN}$ is detected.

To go a step further in the comparison between the molecular line intensity in these kinds of stars, we will now use profile areas corrected for distance. In general, for the same intrinsic luminosity, the observed brightness intensity of sources at different distances from the observer follows a $\sim D^{-2}$ law, where $D$ is the distance. Multiplying the intensities obtained by $D^{2}$, in $\mathrm{kpc}$, we set, in some way, all the stars at the same distance of $1 \mathrm{kpc}$. The distances for the YHGs were taken from de Jager (1998) in the case of IRC +10420 , and from Hipparcos parallax measurements for AFGL 2343, which, despite of its low accuracy, is compatible with the luminosity of a YHG. For the rest of stars the distances are give in Table 1 or were taken from Bujarrabal et al. (1994a).

The result of the distance correction is presented in Fig. 6, for the following representative transitions for each chemistry type: $\mathrm{SiO} J=2-1$ (O-rich) and $J=3-2$, HCN $J=1-0$ and HNC $J=1-0$ (C-rich), all versus ${ }^{13} \mathrm{CO}$. We found, in order of increasing line intensities, first the AGBs, then PPNe, and finally the YHGs. This difference is higher in the case of $\mathrm{SiO}$, an O-bearing molecule, than for $\mathrm{HCN}$ and $\mathrm{HNC}$, which are C-bearing. The reason for this behavior is that, as we have seen, the YHGs show O-rich chemistry.

We note that, although the use of distance corrected areas shows a difference between YHGs and the other objects, it depends directly on the quality of the distance determination. If, as claimed by Josselin \& Lèbre (2001), the distance for AFGL 2343 is smaller than that used here, it would lay in the AGB or PPN region of the diagrams in Fig. 6.

\subsection{Line widths}

The equivalent velocity width, $\Delta V$, provides an independent comparison between YHGs, AGBs and PPNe. We have used as 
Table 2. Peak $T_{\mathrm{mb}}$, rms noise, and integrated area from our observations. The resolution is $2 \mathrm{MHz}$ for the $1 \mathrm{~mm}$ and $2 \mathrm{~mm}$ data, and $1 \mathrm{MHz}$ for the $3 \mathrm{~mm}$ data. ${ }^{*}: 2 \mathrm{MHz}$ resolution. ${ }^{* *}$ : $4 \mathrm{MHz}$ resolution. $\sim$ : Tentative detection. ${ }^{13} \mathrm{CO}$ data from Bujarrabal et al. (2001).

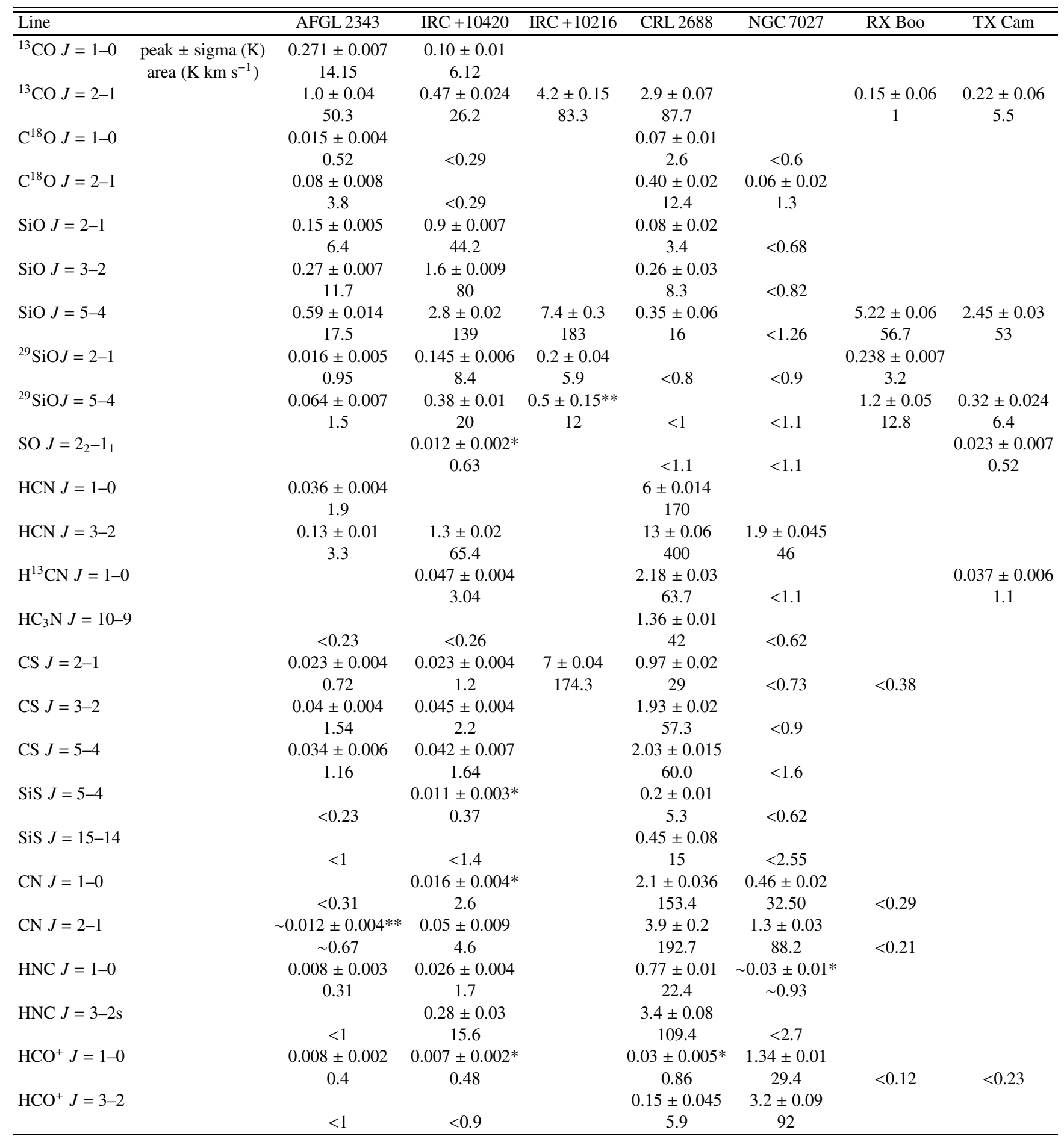

reference line ${ }^{13} \mathrm{CO} J=1-0$, for which we measured $\Delta V$ for all objects.

The mean values found are:

AGBs: $\Delta V=(22 \pm 9) \mathrm{km} \mathrm{s}^{-1}$.

PPNe: $\Delta V=(29 \pm 9) \mathrm{km} \mathrm{s}^{-1}$.

YHGs: $\Delta V=(53 \pm 3) \mathrm{km} \mathrm{s}^{-1}$.

In Fig. 7 we show a histogram of the obtained equivalent line width. This statistical approach to $\Delta V$ in these evolved objects signalizes a major difference between YHGs and AGBs and PPNe. Note that this comparison is distance independent. This result gives us another reason to claim that AFGL 2343 is probably a YHG.

Due to the lack of data for the RSGs we can not obtain an estimate of the equivalent width using this method. From ${ }^{12} \mathrm{CO} J=$ 2-1 line data (Cernicharo et al. 1997) a main value of $55 \mathrm{~km} \mathrm{~s}^{-1}$ is found for RSGs, similar to that of YHGs. This is a confirmation of the evolutionary connection between these objects (see e.g. de Jager 1998).

Note that, for many PPNe, the lines show a core (region with low velocity) and wings (high velocity). The emission in the 


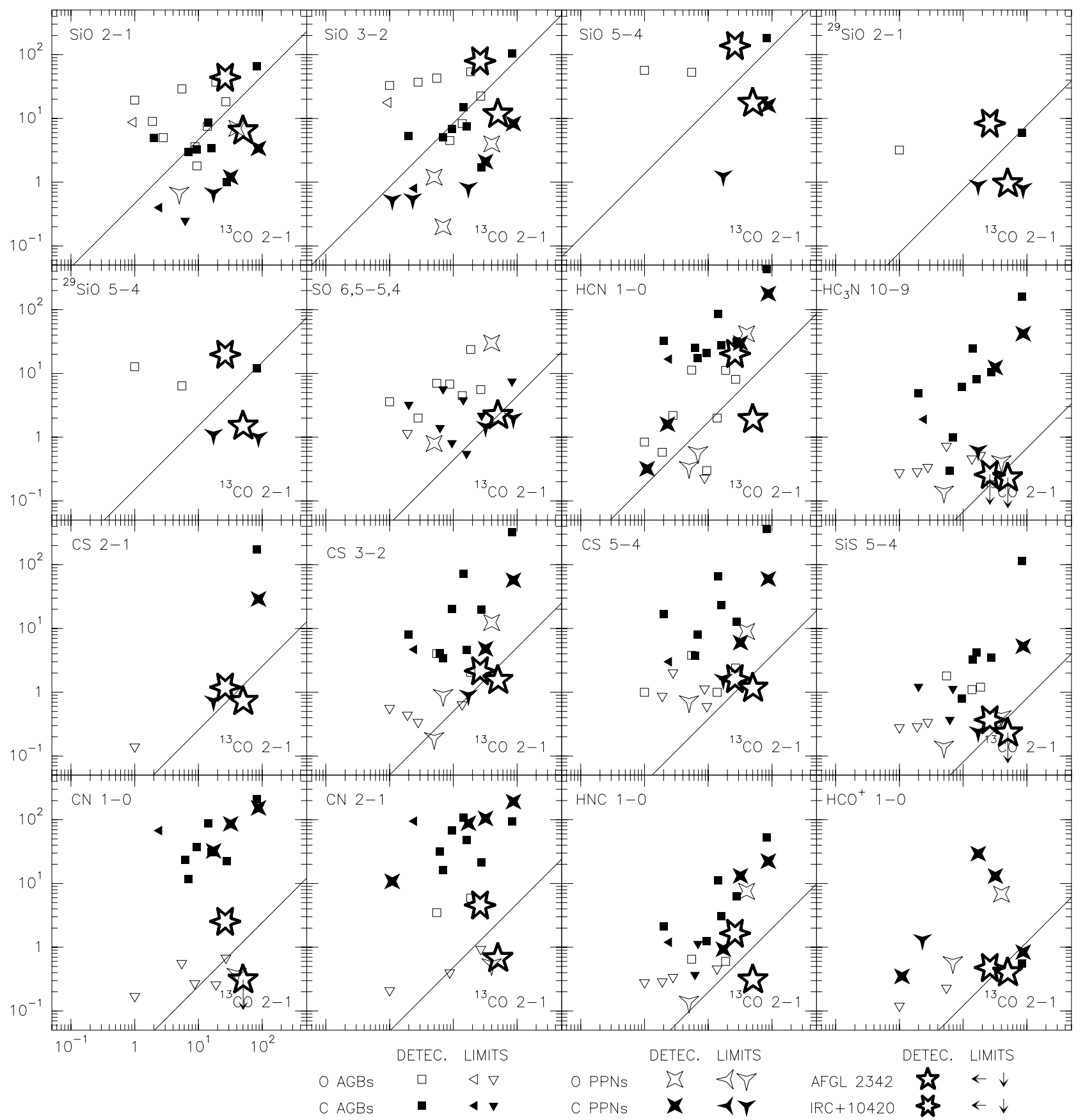

Fig. 3. Profile area diagrams showing some representative line transitions versus ${ }^{13} \mathrm{CO} J=2-1$.

profile wings comes from regions with a high expansion velocity, even larger than $100 \mathrm{~km} \mathrm{~s}^{-1}$ (see e.g. Bujarrabal et al. 2001), but weaker than that of the profile core. The equivalent width is therefore dominated by the core region and it is not very large. In any case, the shape of the line profiles in PPNe is very different from that of AFGL 2343 and IRC+ 10420, which do not show wings (see Figs. 1 and 2).

\section{Abundances}

\subsection{Abundance estimate method}

The formulation used to calculate the abundances from the measured intensities is similar to that used by Bujarrabal et al. (2001). We suppose that the populations of all the rotational levels can be described by a single temperature $T_{\text {rot }}$. We do not assume optically thin emission. The abundance of a molecule can be estimated from the equation:

$$
\begin{aligned}
X= & \ln \left[\frac{1}{1-\frac{T_{\mathrm{mb}}}{S\left(T_{\mathrm{rot}}\right)} \frac{\Omega_{\mathrm{B}}}{\Omega_{\mathrm{S}}}}\right] \frac{8 \pi v_{\mathrm{o}}^{3} Q}{c^{3} g_{\mathrm{u}} A} \frac{\mathrm{e}^{E_{1} / k T_{\mathrm{rot}}}}{\left(1-\mathrm{e}^{\left.-h v_{\mathrm{o}} / k T_{\mathrm{rot}}\right)}\right.} \\
& \times \frac{\Omega_{\mathrm{S}} D^{2} m\left(\mathrm{H}_{2}\right)}{M} \Delta V,
\end{aligned}
$$

where $X$ is the fractional abundance, $Q$ is the partition function, $\Omega_{\mathrm{B}}$ is the HPBW beam size, $\Omega_{\mathrm{S}}$ is the size of the source, $\Delta V$ is the equivalent line width of the emission (in $\mathrm{cm} \mathrm{s}^{-1}$ ), $g_{\mathrm{u}}$ is the degeneration of the upper state, $A$ is the Einstein coefficient, $E_{1}$ is the energy of the lower state of the transition, and $M$ is the mass 


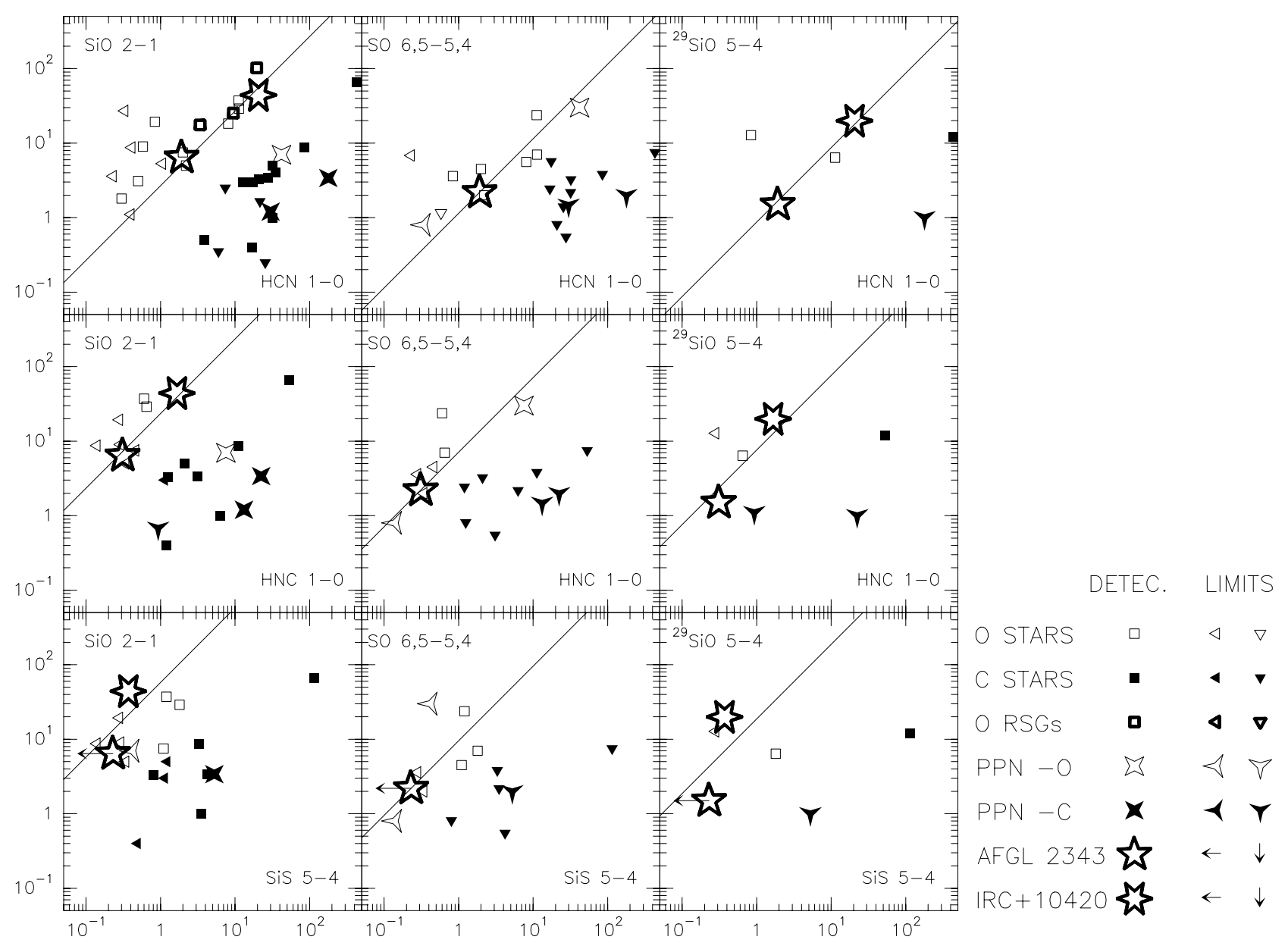

Fig. 4. Profile area diagrams for some pairs of transitions of O-rich molecules versus some of C-rich ones.

of the emitting gas. For linear molecules, $E_{1}=h B_{0} J_{1}\left(J_{1}+1\right)$, where $B_{0}$ is the rotational constant. Note that, in general, the source is much smaller than the beam and the lines are optically thin, therefore the abundance does not depend on $\Omega_{\mathrm{S}}$.

In Eq. (1) the logarithm term appears to account for optically thick emission. However this approximation is only useful for moderate opacities, $\tau$. For values of the optical depth larger than $\sim 2$, the results become too strongly affected by observational and model uncertainties. In other words, for high $\tau$, the emission will only come from the external layers of the envelope, since we are unable to detect the emission from the inner zones, and the derived abundance will just be a lower limit. So, when our calculations indicate an optical depth larger than 2 , we just give the value corresponding to $\tau=2$ as the lower limit to the abundance (this happens for all the transitions of $\mathrm{SiO},{ }^{29} \mathrm{SiO}$, and $\mathrm{HCN}$ in IRC +10420). We also note that this expression becomes merely approximate for $\tau \gtrsim 1$, since we use mean values of $\tau$ within the profile to avoid the integral in the observed velocity.

Due to the low values of $T_{\text {rot }}$ we found (see below), we decided to numerically calculate the partition function with high accuracy, rather than to use approximations. The sum was pursued until the next term had reached a very low tolerance level. In the case of SO, $Q$ was calculated using the energy levels given by Omont et al. (1993).
$\mathrm{CN}$ has fine and hyperfine structure (see Figs. 1 and 2). In order to simplify the calculation of $Q$, we reduce this case to a two $N$-level system by recombination of their Einstein coefficients.

$A_{\mathrm{u} 1}=\frac{1}{\sum_{u} g_{u}} \sum_{\alpha^{\prime}, \alpha} g_{u} A_{u \alpha^{\prime}, l \alpha}$.

This procedure can be safely used only for optically thin emission, where the relative intensity of each line is proportional to the Einstein coefficient, $A$.

The radius of the molecule-rich nebulae around AFGL 2343 and IRC +10420 are taken from results from interferometric CO maps by Castro-Carrizo et al. (2007). We will assume that the ${ }^{13} \mathrm{CO}$ emission extends as far as ${ }^{12} \mathrm{CO}$, while the emission from the rest of the molecules is supposed to come from the densest part of the shell. In fact, in the case of IRC +10420 , the $\mathrm{SiO}$ emission is known to appear in this dense region (Castro-Carrizo et al. 2001). The mass of the emitting region is also derived from the results obtained by Castro-Carrizo et al. (2007). These data are summarized in Table 3.

We estimate $T_{\text {rot }}$ from the line intensity ratio for the molecules in which we have observed more than one transition. Sometimes, the value of $T_{\text {rot }}$ found in this way was slightly lower than the observed brightness temperature, after correcting the observed $T_{\mathrm{mb}}$ for the dilution factor, $\Omega_{\mathrm{S}} / \Omega_{\mathrm{B}}$. In these cases, we imposed $T_{\text {rot }}$ to be equal to the brightness temperature.

For the molecules with only one observed transition or with only one detection, we took an average rotational temperature, 


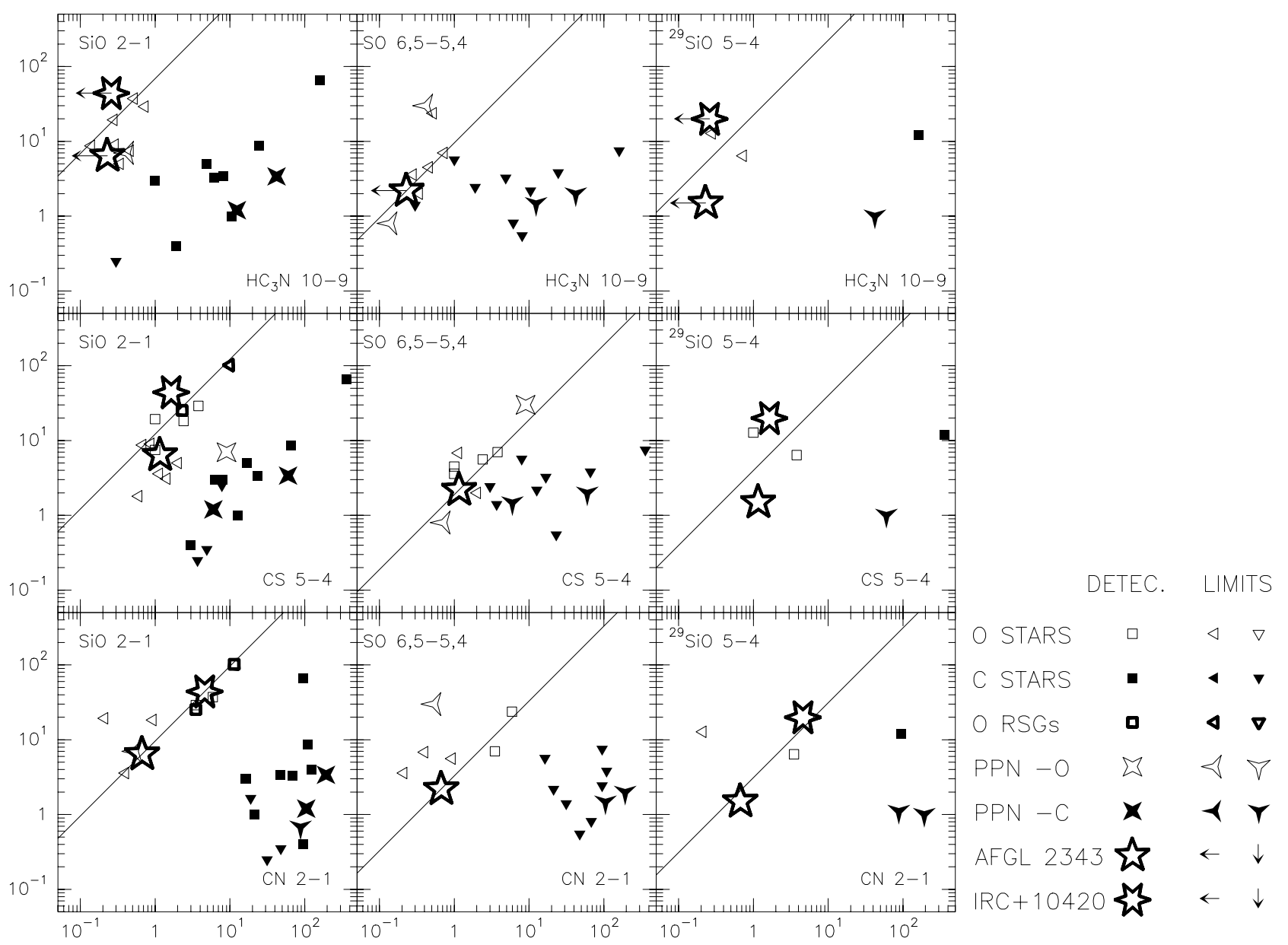

Fig. 5. Same as Fig. 4 for other pairs.

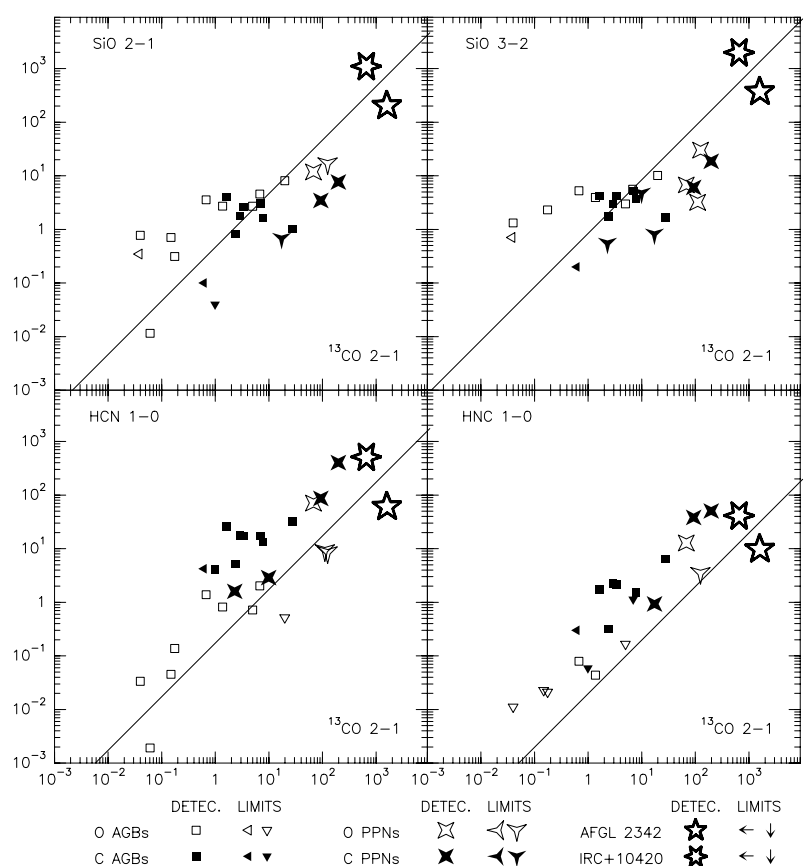

Fig. 6. Distance-corrected profile areas diagram for some representative lines versus ${ }^{13} \mathrm{CO} J=2-1$.

which was found to be $\sim 10 \mathrm{~K}$ for AFGL 2343 and $\sim 11 \mathrm{~K}$ for IRC +10420 . In some cases, where we have a limit for the

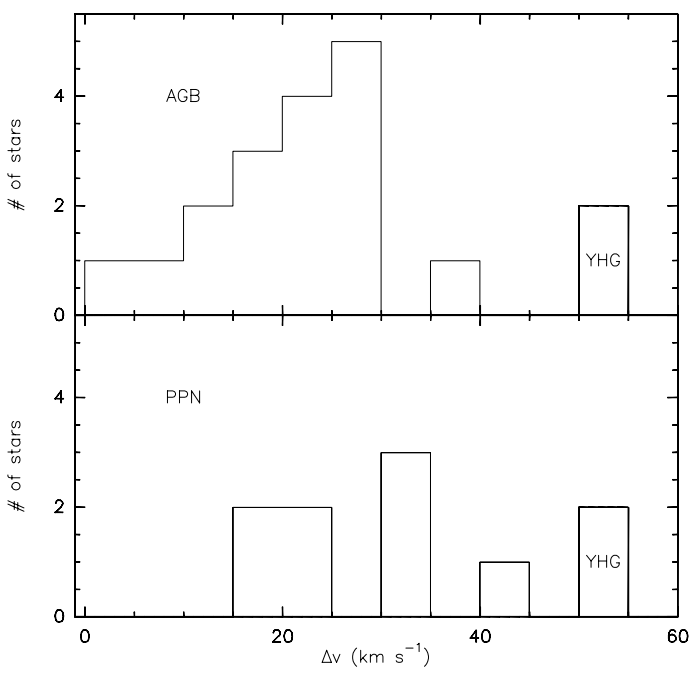

Fig. 7. Histogram of the profile equivalent line width. Upper panel shows the comparison between AGBs and YHGs. Lower panel refers to PPNe versus YHGs.

transition with the highest $J$, we obtained that the upper limit to the abundance from the non-detection is lower than the abundance derived from the detected line, using the mean $T_{\text {rot. }}$. In this case, we decrease the rotational temperature until both values of the abundances derived are equal, being an upper limit for $T_{\text {rot }}$. 


\subsection{Abundance results}

We present the results of our abundance calculations in Table 4. We also show the derived rotational temperatures. The temperatures are systematically higher for the molecules that show high optical depths, as expected when the excitation temperatures are lower than the kinetic temperature.

We deduce the mean densities for the $\mathrm{CO}$ emitting region from the data in Table 3: $n \sim 5 \times 10^{3} \mathrm{~cm}^{-3}$ for AFGL 2343, and $n \sim 8 \times 10^{2} \mathrm{~cm}^{-3}$ for IRC +10420 . The mean densities derived for the emitting regions of the other molecules are $n \sim 4 \times 10^{4} \mathrm{~cm}^{-3}$ for AFGL 2343 and $n \sim 2 \times 10^{4} \mathrm{~cm}^{-3}$ for IRC +10420 . These relatively low densities are compatible with the idea that most lines, except in particular those of $\mathrm{CO}$, should be underexcited.

AFGL 2343 shows a general underabundance in all the molecules but ${ }^{13} \mathrm{CO}$. The abundances of molecules other than ${ }^{13} \mathrm{CO}$ are in general more than ten times higher in IRC +10420 than in AFGL 2343. AFGL 2343 shows an abundance of ${ }^{13} \mathrm{CO}$ comparable to that typical of AGB stars, while this abundance in IRC +10420 is higher by a factor 2 .

The emission of molecules in AFGL 2343, apart from ${ }^{13} \mathrm{CO}$, could come from a region smaller than that assumed here (Sect. 4.1), containing significantly less mass. This would result in an increase in the derived molecular abundances (in that region) for AFGL 2343. In fact the emission of such species in AGB envelopes usually comes from regions at a distance from the star of several $10^{16} \mathrm{~cm}$, although the $\mathrm{SiO}$ emission from IRC +10420 is confirmed to appear at a distance of about $10^{17} \mathrm{~cm}$. We note the difference in the assumed radii of the dense region for both stars (see Table 3), being that of AFGL 2343 twice that of IRC +10420 .

\subsection{Comparison with previously published results}

With the results in Table 4, we are able to compare the abundances here calculated with other published results for YHGs, like those obtained in Bujarrabal et al. (1994a) and Bachiller et al. (1997b). In these papers the distance assumed for IRC +10420 is $3.4 \mathrm{kpc}$ and for AFGL 2343 is $6 \mathrm{kpc}$. To compare the results of the abundance calculations first we must correct for the effects of the different assumed distances.

The average difference is $\sim 20$ for IRC +10420 and around $\sim 300$ for AFGL 2343. In order to find out the origin of these discrepancies we must focus on the assumptions made in the different calculations. Both Bujarrabal et al. (1994a) and Bachiller et al. (1997b) used the same method, assuming optically thin emission. In fact, Eq. (1), here used to calculate abundances, would become that used by Bujarrabal et al. (1994a,b) and Bachiller et al. (1997b) if we assume, as in those papers, optically thin emission and a negligible background temperature $\left(T_{\mathrm{bg}} \sim 0\right)$. For those cases with optically thin emission, the differences in the calculations can only be due to the assumed values of the emitting mass and of $T_{\text {rot }}$. For optically thick cases, the differences also depend on the source size and opacity.

In Table 5 we can see the radii used and the masses obtained for the emitting regions by Bujarrabal et al. (1994a) and Bachiller et al. (1997b). The radii assumed by these authors for regions emitting in molecules other than $\mathrm{CO}$ in YHGs are significantly lower than the values obtained from PdB maps (Sect. 3), leading to smaller masses and, therefore, to higher abundances. Indeed, the discrepancies found in the abundances are almost fully explained by these differences in the masses adopted for the emitting region.
Table 3. Assumed radii and mass of the emitting regions, based on the ${ }^{12} \mathrm{CO}$ data from Castro-Carrizo et al. (2007).

\begin{tabular}{l|ccc|ccc}
\hline \hline Molecule & $R_{\text {in }}(\mathrm{cm})$ & $R_{\text {out }}(\mathrm{cm})$ & $M\left(M_{\odot}\right)$ & $R_{\text {in }}(\mathrm{cm})$ & $R_{\text {out }}(\mathrm{cm})$ & $M\left(M_{\odot}\right)$ \\
\hline & \multicolumn{3}{|c|}{ AFGL 2343 } & \multicolumn{3}{|c}{ IRC +10420} \\
\hline${ }^{13} \mathrm{CO}$ & $1 \times 10^{15}$ & $5 \times 10^{17}$ & 4.02 & $3 \times 10^{16}$ & $5.2 \times 10^{17}$ & 0.7 \\
Others & $1 \times 10^{15}$ & $2.5 \times 10^{17}$ & 3.31 & $3 \times 10^{16}$ & $1.24 \times 10^{17}$ & 0.24 \\
\hline
\end{tabular}

Table 4. Mean abundances and rotational temperatures derived for AFGL 2343 and IRC +10420. ${ }^{1}$ : forced to be equal to the average $T_{\text {rot }}$ value. ${ }^{2}: T_{\text {rot }}$ imposed to be equal to brightness temperature. ${ }^{3}$ : from intensity upper limit; see text for details.

\begin{tabular}{l|cc|cc}
\hline \hline Line & $\langle X\rangle$ (AFGL 2343) & $T_{\text {rot }}(\mathrm{K})$ & $\langle X\rangle($ IRC +10420$)$ & $T_{\text {rot }}(\mathrm{K})$ \\
\hline${ }^{13} \mathrm{CO}$ & $2.6 \times 10^{-5}$ & 9 & $4.7 \times 10^{-5}$ & 10 \\
$\mathrm{C}^{18} \mathrm{O}$ & $1.5 \times 10^{-6}$ & 20 & $<6.6 \times 10^{-6}$ & $11^{1}$ \\
$\mathrm{CN}$ & $3.6 \times 10^{-9}$ & 6 & $3.8 \times 10^{-7}$ & 6 \\
$\mathrm{CS}$ & $8.3 \times 10^{-9}$ & 10 & $1.9 \times 10^{-7}$ & 9 \\
$\mathrm{H}^{13} \mathrm{CN}$ & & & $2.9 \times 10^{-7}$ & $11^{1}$ \\
$\mathrm{HC}_{3} \mathrm{~N}$ & $5.5 \times 10^{-9}$ & $10^{1}$ & $5.7 \times 10^{-8}$ & $11^{1}$ \\
$\mathrm{HCN}$ & $1.1 \times 10^{-8}$ & 7 & $>2.2 \times 10^{-6}$ & $36^{2}$ \\
$\mathrm{HNC}$ & $2.0 \times 10^{-9}$ & $\leq 8^{3}$ & $1.6 \times 10^{-7}$ & 18 \\
$\mathrm{HCO}$ & $2.0 \times 10^{-9}$ & $\leq 7^{3}$ & $2.7 \times 10^{-8}$ & $\leq 6^{3}$ \\
$\mathrm{SiO}$ & $5.4 \times 10^{-8}$ & 11 & $>1.3 \times 10^{-5}$ & $79^{2}$ \\
$\mathrm{SiS}$ & $8.5 \times 10^{-9}$ & $10^{1}$ & $1.5 \times 10^{-7}$ & $11^{1}$ \\
$\mathrm{SO}$ & & & $1.6 \times 10^{-6}$ & 11 \\
${ }^{29} \mathrm{SiO}$ & $6.9 \times 10^{-9}$ & 8 & $>1.0 \times 10^{-6}$ & $15^{2}$ \\
\hline
\end{tabular}

Table 5. Radii and mass of the emitting gas for each molecule used in Bujarrabal et al. (1994a) and in Bachiller et al. (1997b). *: Shell, $R_{\text {in }}=3.1 \times 10^{16} \mathrm{~cm}$.

\begin{tabular}{l|ll|ll}
\hline \hline Molecule & $R_{\text {out }}(\mathrm{cm})$ & $M\left(M_{\odot}\right)$ & $R_{\text {out }}(\mathrm{cm})$ & $M\left(M_{\odot}\right)$ \\
\hline & AFGL 2343 & & IRC+10420 & \\
\hline${ }^{13} \mathrm{CO}$ & $10^{17}$ & 0.2 & $10^{17}$ & 0.06 \\
$\mathrm{SiO}$ & $2 \times 10^{15}$ & $4.2 \times 10^{-3}$ & $2 \times 10^{15}$ & $1.2 \times 10^{-3}$ \\
$\mathrm{CN}$ & & & $1.1 \times 10^{17 *}$ & 0.05 \\
Other & $10^{16}$ & 0.02 & $10^{16}$ & $6 \times 10^{-3}$ \\
\hline
\end{tabular}

Another important factor in the abundance estimate is the rotational temperature. In Bujarrabal et al. (1994a,b), a $T_{\text {rot }}$ of $20 \mathrm{~K}$ is assumed. The temperatures we found here are in most of the cases lower than $20 \mathrm{~K}$. Bachiller et al. (1997b), however, estimate the rotational temperature from the ratio between $\mathrm{CN} N=$ $2-1$ and $N=1-0$ emission, and it is similar to the one obtained here for this molecular transition.

In some cases, the ratio between the abundances calculated here and those from the cited authors is lower than the ratio of the masses. This is explained by the difference in $T_{\text {rot }}$. Lower rotational temperatures lead to higher abundances in the case of transitions with a high $J$, like in the case of CS $J=3-2$ and $J=$ $5-4$, which were the transitions used to calculate the abundance in Bujarrabal et al. (1994a).

\subsection{Comparison with abundances in AGB stars}

The relative abundances, $X$, determined here (see Table 4) must be compared with the standard abundances found in circumstellar envelopes (e.g. Bujarrabal et al. 1994a), in order to understand the differences between our YHGs and those.

The values of $X$ found for IRC +10420 are in full agreement with those supposed for the O-rich CSEs, except for the high abundances obtained for ${ }^{13} \mathrm{CO}$ and $\mathrm{HCN}$. In the case of ${ }^{13} \mathrm{CO}$, 
this overabundance is a factor 2. Note that the value of $X_{\mathrm{HCN}}$ is affected by the opacity, as said in Sect. 4.1, therefore low abundances were found by Bujarrabal et al. (1994), who assumed low optical depths.

In AFGL 2343, except for ${ }^{13} \mathrm{CO}$, the abundances are much lower (around 70 times on average) than the standard values for the CSEs around AGBs. As we mentioned in Sect. 4.2, such low abundances found in AFGL 2343 could be related to the extent of the circumstellar layer emitting in these molecular lines. In AFGL 2343, the region we assumed to be molecule rich is as extended as $2.5 \times 10^{17} \mathrm{~cm}$, but there is no observational confirmation of such a large extent. If the region at which molecules other than $\mathrm{CO}$ are abundant in this source is comparable to that of IRC +10420 , then the emitting mass would be much smaller than the mass assumed in our abundance calculation $\left(\sim 0.05 M_{\odot}\right.$, see mass estimates by Castro-Carrizo et al. 2007). This would yield molecular abundances in this region comparable to those usually found in molecule rich CSEs.

\section{Conclusions}

We observed several molecular transitions of a wide variety of species (see Sect. 2) in the yellow hypergiants (YHGs) AFGL 2343 and IRC +10420 as well as in several AGB stars and PPNe. Previously published observations were added to our sample, to obtain a better comparison between the properties of the different objects.

From the collected spectra, we have compared the ratios of the integrated area for several relevant pairs of lines in the YHGs with those in AGBs and PPNe. We find that the YHGs studied here show, as expected, O-rich chemistry. However, the emission of molecules other than ${ }^{13} \mathrm{CO}$ in AFGL 2343 is significantly weaker than that found for the O-rich AGBs and for IRC +10420 .

The comparison of the ratios of the integrated profile areas corrected by distance has shown a clear difference between YHGs, whose emission is the more intense, and PPNe and AGB stars. Another difference, distance independent, appears when comparing equivalent line widths, which are much higher for YHGs than for the other evolved objects. At this respect, we note the absence of wings in the line profiles of AFGL 2343 and IRC +10420 .

All this supports the idea that AFGL 2343 is a YHG rather than a PPN, as also deduced from CO maps (Castro-Carrizo et al. 2007).

We estimated the abundances of the different molecular species within the circumstellar envelopes of AFGL 2343 and IRC +10420 using the method described in Sect. 4.1. We assumed that molecular emission, apart from $\mathrm{CO}$ and its isotopes, comes from the innermost dense region found by Castro-Carrizo et al. (2007) and described in Table 3, which extends to several $10^{17} \mathrm{~cm}$. Note that this extent is larger than that usually found for the same molecules in AGB stars. Dust shielding would prevent photodissociation of these molecules in the further regions assumed for the YHGs. On the other hand, ${ }^{13} \mathrm{CO}$ line emission in AGB CSEs and PPNe usually extends as much as ${ }^{12} \mathrm{CO}$ emission; we recall that the ${ }^{12} \mathrm{CO}$ envelope in our sources has been mapped by Castro-Carrizo et al. (2007).

The abundance found for ${ }^{13} \mathrm{CO}$ in AFGL 2343 is comparable with that usual for AGBs, while that of IRC +10420 is larger by a factor of 2 . The abundances of other molecules found in IRC +10420 are comparable, in general, to those of O-rich AGB stars, but those of AFGL 2343 are found to be around 40 times lower in average. These low abundances could be due to the fact that these molecules are in fact abundant only in an inner region with a relatively low mass.

Acknowledgements. This work has been supported by the Spanish Ministerio de Ciencia y Tecnologia and European FEDER funds, under grants AYA2003-7584 and ESP2003-04957. The contribution of AC-C was supported by the 6th European Community Framework Programme through a Marie-Curie IntraEuropean Fellowship.

\section{References}

Bachiller, R., Forveille, T., Huggins, P. J., \& Cox, P. 1997a, A\&A, 324, 1123 Bachiller, R., Fuente, A., Bujarrabal, V., et al. 1997b, A\&A, 319, 235

Bujarrabal, V., Alcolea, J., \& Planesas, P. 1992, A\&A, 257, 701B

Bujarrabal, V., Fuente, A., \& Omont, A. 1994a, A\&A, 285, 247

Bujarrabal, V., Fuente, A., \& Omont, A. 1994b, ApJ, 421, L47

Bujarrabal, V., Castro-Carrizo, A., Alcolea, J., \& Sánchez Contreras, C. 2001, A\&A, 377, 868

Castro-Carrizo, A., Lucas, R., Bujarrabal, V., Colomer, F., \& Alcolea, J. 2001, A\&A, 368, L34

Castro-Carrizo, A., Quintana-Lacaci, G., Bujarrabal, V., Neri, R., \& Alcolea, J. 2007, A\&A, 465, 457

Cernicharo, J., Alcolea, J., Baundry, A., et al. 1997, A\&A, 319, 607

de Jager, C. 1998, ARA\&A, 8, 145

Hawkins, G. W., Skinner, C. J., Meixner, M., et al. 1995, ApJ, 452, 314

Humphreys, R. M. 1991, in Wolf Rayet Stars, ed. K. A. van der Hucht, \& B. Hidayat (Kluwer), 485

Humphreys, R. M., Smith, N., Davidson, K., et al. 1997, ApJ, 114, 2778

Humphreys, R. M., Davidson, K., \& Smith, N. 2002, ApJ, 124, 1026

Jones, T. J., Humphreys, R. M., Gehrz, R. D., et al. 1993, ApJ, 411, 323

Josselin, E., \& Lèbre, A. 2001, A\&A, 367, 826

Jourdain de Muizon, M., Cox, P., \& Lequeux, J. 1990, A\&AS, 83, 337

Klochkova, V. G., Chentsov, E. L., \& Panchuk, V. E. 1997, MNRAS, 292, 19

Likkel, L. 1989, ApJ, 344, 350

Lucas, R., \& Guélin, M. 1990, in Submillimetre Astronomy, ed. G. D. Watt, \& A. S. Webster (Kluwer), 97

Maeder, A., \& Meynet, G. 1988, A\&AS, 76, 411

Mauersberger, R., Guélin, M., Martín-Pintado, J., et al. 1989, A\&AS, 79, 217

Meixner, M., Ueta, J., Dayal, A., et al. 1999, ApJS, 122, 221

Molster, F. J., Waters, L. B. F. M., Tielens, A. G. G. M., \& Barlow, M. J. 2002, A\&A, 382, 184

Neri, R., Kahane, C., Lucas, R., Bujarrabal, V., \& Loup, C. 1998, A\&AS, 130, 1

Omont, A., Lucas, R., Morris, M., \& Guilloteau, S. 1993, A\&A, 267, 490

Oudmaijer, R. D., Groenewegen, M. A. T., Matthews, H. E., et al. 1996, MNRAS, 280, 1062

Oudmaijer, R. D. 1998, A\&AS, 129, 541

Sánchez Contreras, C., Bujarrabal, V., \& Alcolea, J. 1997, A\&A, 327, 689

Skinner, C. J., Meixner, M., \& Bobrowsky, M. 1998, MNRAS, 300, 29

Reid, M. J., Moran, J. M., Leach, R. W., et al. 1979, ApJ, 227, L89

Thévenin, F., Parthasarathy, M., \& Jasniewicz, G. 2000, A\&A, 359, 138 
G. Quintana-Lacaci et al.: Chemistry in the CSEs around YHGs, Online Material $p 1$

\section{Online Material}


G. Quintana-Lacaci et al.: Chemistry in the CSEs around YHGs, Online Material $p 2$

\section{Appendix A: Spectra of other program stars}
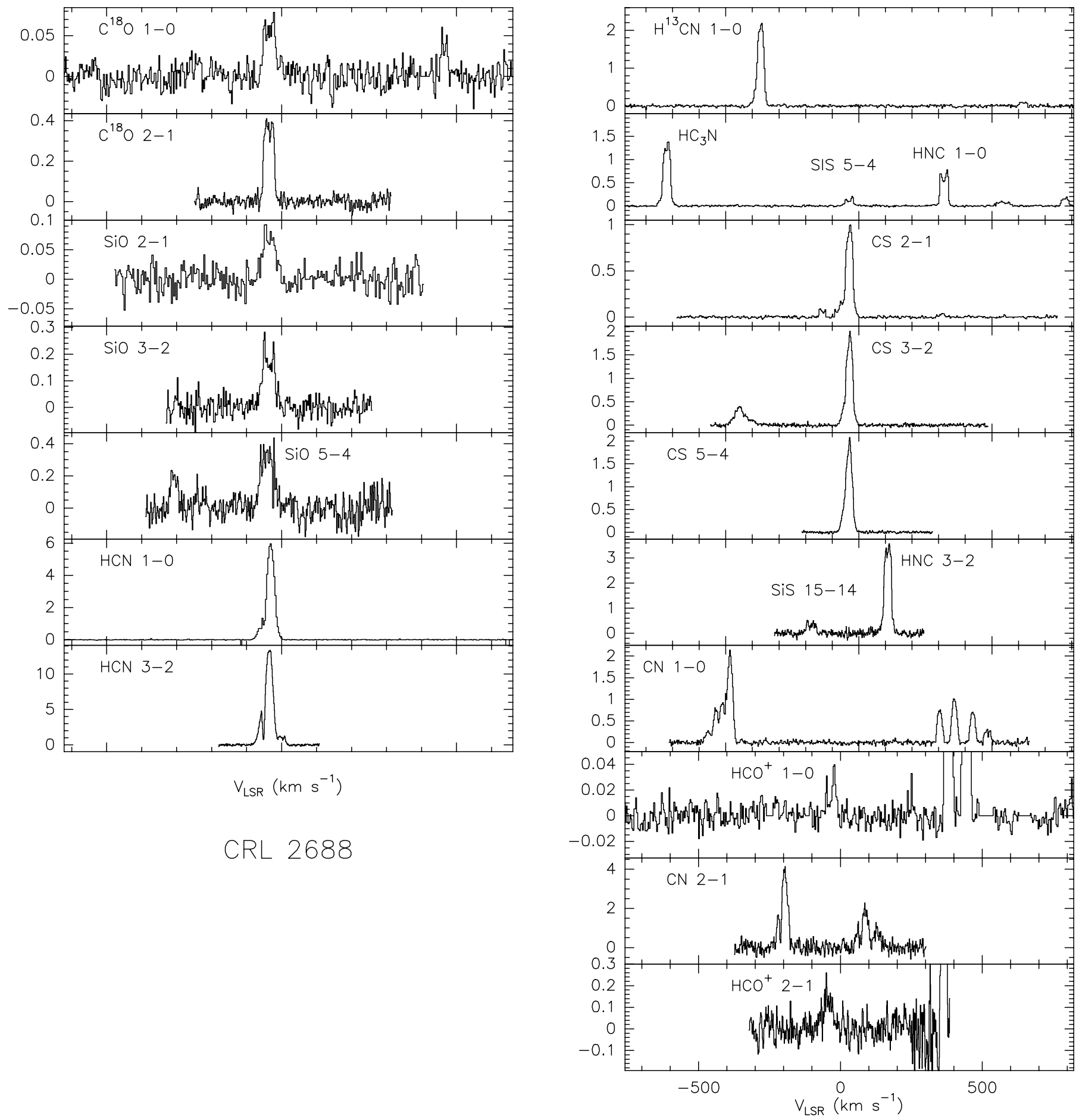

Fig. A.1. Observed spectra towards CRL 2688. The intensity scale is given in units of main-beam brightness temperature (K). 
G. Quintana-Lacaci et al.: Chemistry in the CSEs around YHGs, Online Material p 3

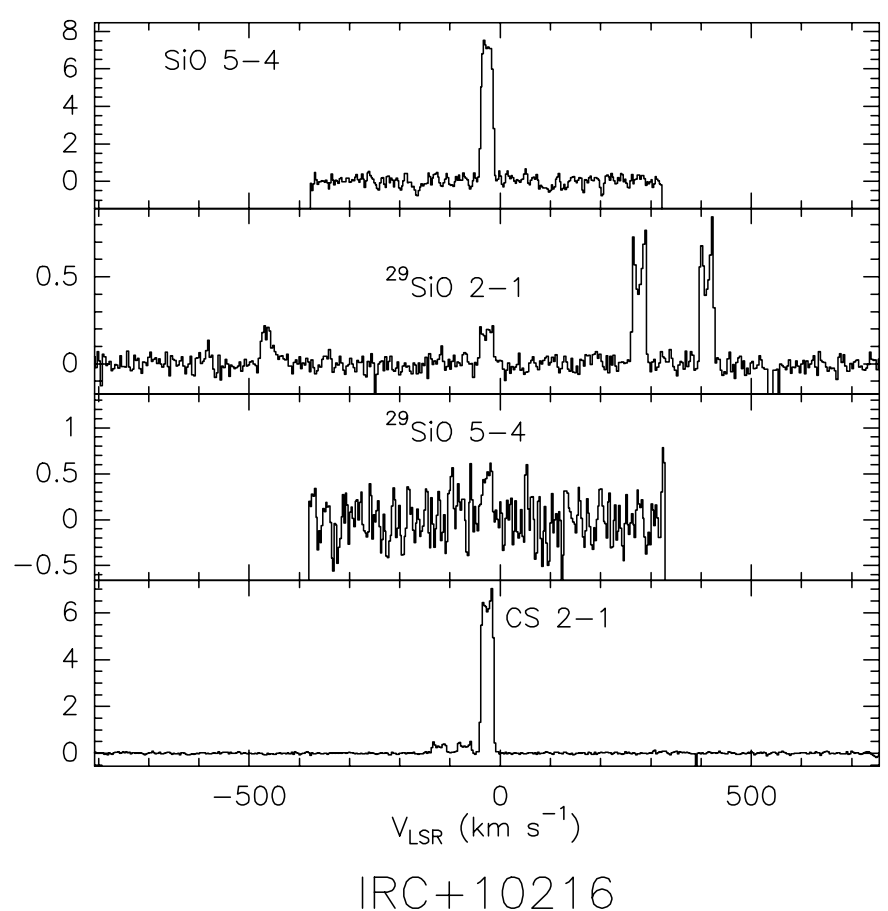

Fig. A.2. Observed spectra towards IRC +10216 . The intensity scale is given in units of main-beam brightness temperature $(\mathrm{K})$.

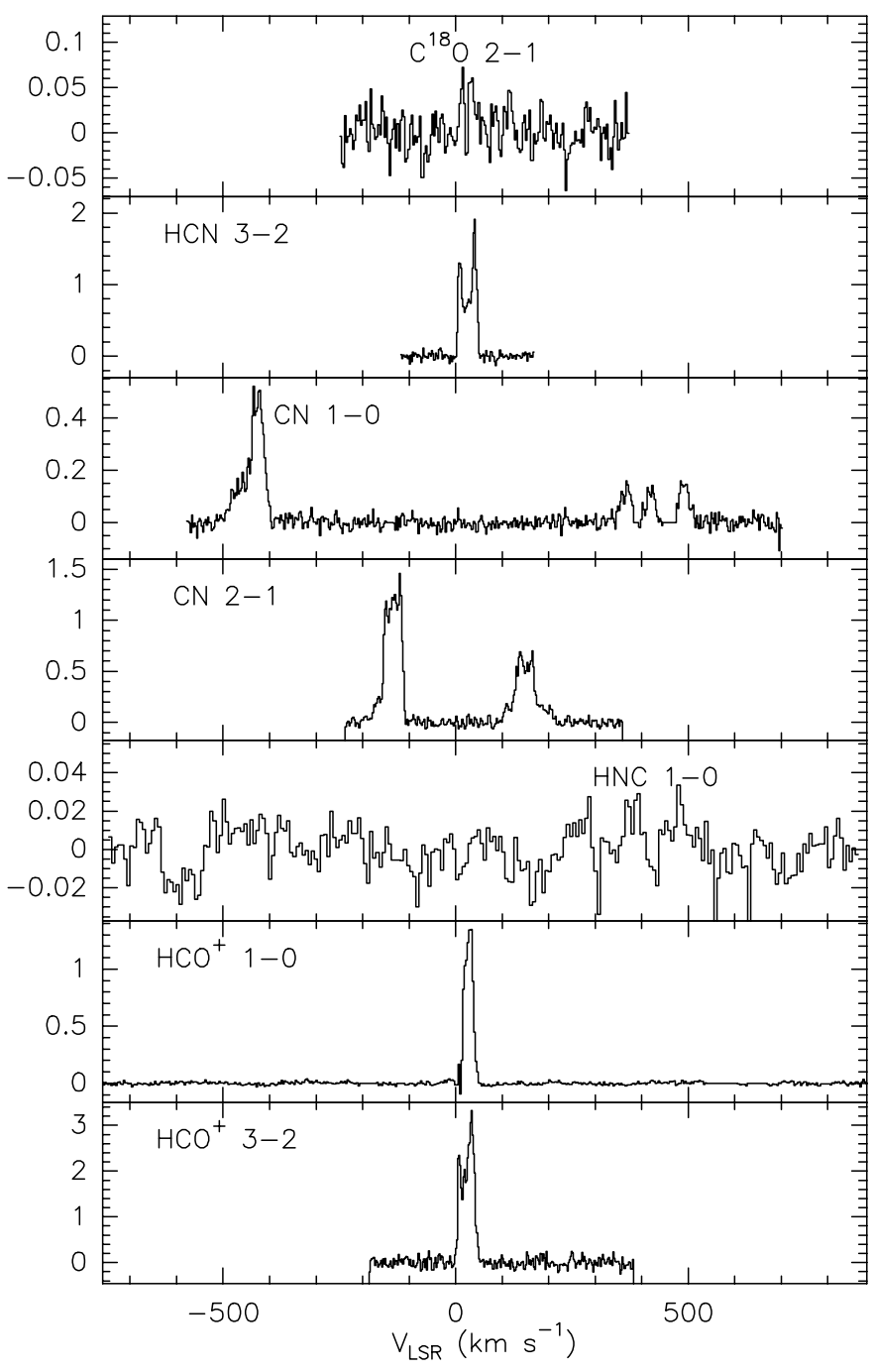

\section{NGC 7027}

Fig. A.3. Observed spectra towards NGC 7027. The intensity scale is given in units of main-beam brightness temperature (K).

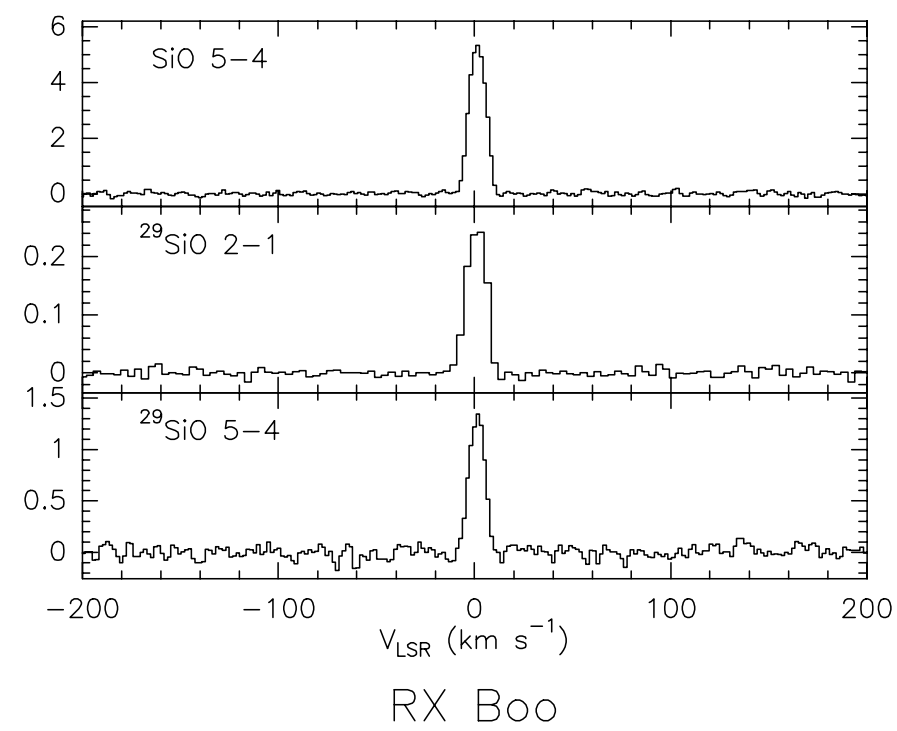

Fig. A.4. Observed spectra towards RX Boo. The intensity scale is given in units of main-beam brightness temperature $(\mathrm{K})$. 
G. Quintana-Lacaci et al.: Chemistry in the CSEs around YHGs, Online Material p 4

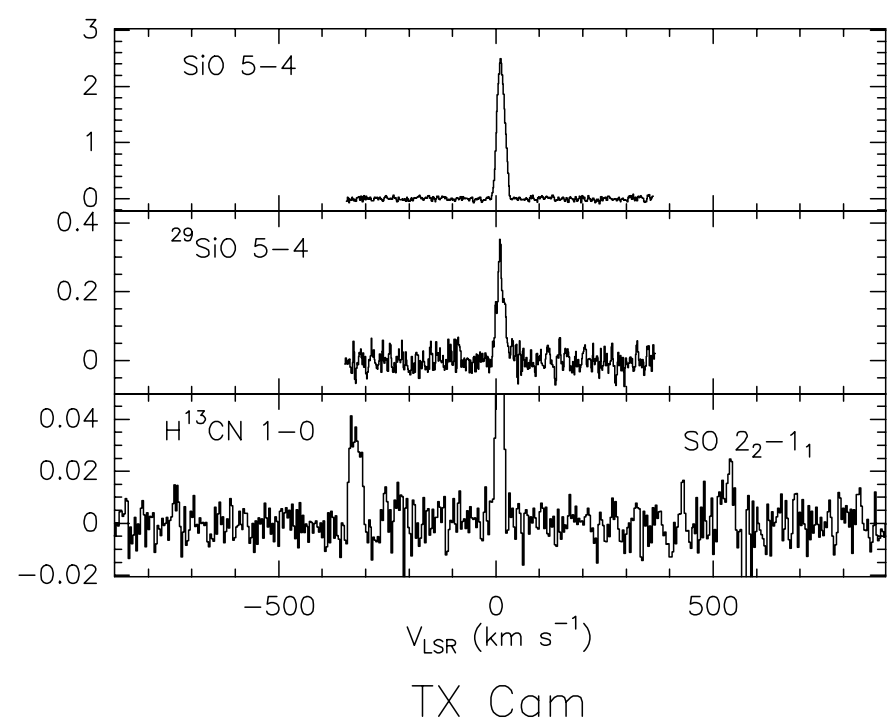

Fig. A.5. Observed spectra towards TX Cam. The intensity scale is given in units of main-beam brightness temperature (K). 